\title{
Standing fast magnetoacoustic kink waves of solar coronal loops with field-aligned flow
}

\author{
M. Gruszecki ${ }^{1}$, K. Murawski ${ }^{1}$, and L. Ofman ${ }^{2}$ \\ ${ }^{1}$ Group of Astrophysics and Gravity Theory, Institute of Physics, UMCS, ul. Radziszewskiego 10, 20-031 Lublin, Poland \\ e-mail: marcingruszecki@wp.pl \\ 2 The Catholic University of America, NASA Goddard Space Flight Centre, Code 671, Greenbelt, MD 20771, USA
}

Received 30 March 2008 / Accepted 6 June 2008

\section{ABSTRACT}

\begin{abstract}
Aims. We refer to the recent observational data of Hinode, which detected weakly-attenuated coronal loop oscillations in the presence of background flow (Ofman \& Wang 2008, A\&A, 482, L9). Vertical loop oscillations that lasted for three wave periods were reported with a wave period $P=113 \pm 2 \mathrm{~s}$, attenuation time $\tau=560 \pm 260 \mathrm{~s}$, and wave amplitude $A_{\max }=0.67 \pm 0.12 \mathrm{Mm}$. Ofman \& Wang (2008) estimated the flow speed within the range of $74-123 \mathrm{~km} \mathrm{~s}^{-1}$. We consider impulsively generated standing fast magnetoacoustic kink waves of a straight solar coronal slab with field-aligned internal flow. We aim to determine the influence of such flow on the spatial and temporal signatures of these waves.

Methods. The time-dependent, ideal magnetohydrodynamic equations are solved numerically.

Results. The numerical results show that as a result of wave scattering on inhomogeneous flow kink waves experience stronger attenuation than for a still plasma, while $P$ remains weakly altered by this flow. Numerically evaluated values of $A_{\max }$ and $P$ are close to the observational data. A value of $\tau$ is about two times smaller than observed.
\end{abstract}

Key words. magnetohydrodynamics (MHD) - Sun: corona - Sun: oscillations

\section{Introduction}

In the frame of coronal seismology (Uchida 1970; Roberts et al. 1984; Nakariakov et al. 1995) solar magnetohydrodynamic (MHD) waves are recognised as an important diagnostic tool for the medium through which they propagate. The recent years have brought observations of a variety of coronal loop oscillations which were interpreted either as propagating or standing MHD waves. The oscillations observed by Aschwanden et al. (1999) are horizontally polarised, while the oscillations detected by Wang \& Solanki (2004) are vertically polarised. Standing (Wang et al. 2002) and propagating (Ofman et al. 1997; DeForest \& Gurman 1998; Ofman et al. 1999; Ofman et al. 2000; De Moortel et al. 2002) slow magnetoacoustic waves were also reported. The method of coronal seismology was successfully applied to these waves and oscillations by Nakariakov \& Verwichte (2005).

This work is stimulated by results of the first Hinode observation of coronal loop oscillations (Ofman \& Wang 2008). The observations were made by Solar Optical Telescope on Hinode with the $\mathrm{Ca}$ II $\mathrm{H}$ line filter, which has the formation temperature of $10^{4}-2 \times 10^{4} \mathrm{~K}$. The loops were traced with thin threads of field aligned flow of chromospheric material in the above temperature range. Co-spatial loops were seen by SOHO Extreme ultraviolet Imaging Telescope in $195 \AA$ at this location and time that corresponds to coronal temperature (peak formation temperature of 1.6 MK), but with much lower spatial and temporal resolution than Hinode/SOT (Ofman \& Wang 2008). The observational data was interpreted as the oscillations that were seen for about 3 wave periods, with a wave period lasting about 2 min. These oscillations occurred in the presence of background flow whose magnitude was estimated in the range of $74-123 \mathrm{~km} \mathrm{~s}^{-1}$.
The effect of flow on magnetoacoustic waves was discussed in the literature. For instance, Nakariakov \& Roberts (1995) considered steady flows of plasma using slab geometry. They showed that flows change the characteristics of magnetoacoustic modes qualitatively and quantitatively. They concluded that flow breaks the symmetry (e.g., by the appearance of a new type of trapped waves, namely backward waves). Terra-Hommem et al. (2003) extended the work of Nakariakov \& Roberts (1995) to cylindrical geometry. It was demonstrated by Joarder et al. (1997) that for the flows slower than the threshold of KelvinHelmholtz instability, backward waves can be of negative energy and hence subject to instabilities associated with negative energy waves. Murawski \& Roberts (1994) developed a model of the solar $f$-mode in a randomly flowing plasma to show that the $f$-mode exhibits frequency reduction and amplitude attenuation. Murawski et al. (2001) showed that random mass density leads to acceleration and attenuation of fast magnetoacoustic waves that propagate perpendicularly to a uniform magnetic field. Grappin et al. (2003) investigated time-dependent siphon flows in coronal loops driven by Alfvén waves. When considering a 1.5-D isothermal MHD model, they showed that the reaction of the loop to Alfvén waves depends entirely on parallel velocity fluctuations. Grappin et al. (2005) using an isothermal MHD model of the solar corona, studied coronal loops reaction to Alfvén waves generated at the base of the corona. They found steady increase of the mass density along the excited loops. They also observed density oscillations along the apex of the longest loops, that is, those along which waves are substantially attenuated. Recently, Soler et al. (2008) inferred that the presence of flow has no effect on the attenuation of slow magnetoacoustic and thermal modes, whereas fast magnetoacoustic kink waves are more (less) attenuated when they propagate parallel (antiparallel) to the flow. 
The above mentioned studies were devoted to propagating waves and the effect of flow on standing waves was not studied so far. We aim to explore the importance of parallel background flow on standing fast magnetoacoustic kink waves by comparing results for two cases: (a) flowing and (b) still plasmas. We realise our goal in the context of the Hinode data (Ofman \& Wang 2008), which revealed a co-existence of standing waves and background flow.

This paper is organised as follows. The numerical model is described in the following section. Numerical results are presented and discussed in Sect. 3. This paper is concluded by a short summary of the main results in Sect. 4.

\section{Numerical model}

We describe coronal plasma with a use of the ideal MHD equations, viz.:

$$
\begin{array}{r}
\frac{\partial \varrho}{\partial t}+\nabla \cdot(\varrho \boldsymbol{V})=0, \\
\varrho \frac{\partial \boldsymbol{V}}{\partial t}+\varrho(\boldsymbol{V} \cdot \nabla) \boldsymbol{V}=-\nabla p+\frac{1}{\mu}(\nabla \times \boldsymbol{B}) \times \boldsymbol{B}, \\
\frac{\partial \boldsymbol{B}}{\partial t}=\nabla \times(\boldsymbol{V} \times \boldsymbol{B}), \\
\frac{\partial p}{\partial t}+\nabla \cdot(p \boldsymbol{V})=(1-\gamma) p \nabla \cdot \boldsymbol{V}, \\
\nabla \cdot \boldsymbol{B}=0, \\
p=\frac{R}{\hat{\mu}} \varrho T .
\end{array}
$$

Here $\varrho$ is mass density, $p$ is gas pressure, $\boldsymbol{V}=\left[V_{x}, V_{y}, V_{z}\right]$ is flow velocity, $\boldsymbol{B}=\left[B_{x}, B_{y}, B_{z}\right]$ is magnetic field, $\mu$ is the magnetic permeability, $\gamma=5 / 3$ is the adiabatic index, $T$ is temperature and $\hat{\mu}$ is the mean atomic weight (average mass per particle in units of mass of a proton). We limit our discussion to a 2-D magnetically structured medium in which the equilibrium magnetic field and flow are polarised in the $x-z$ plane such that $V_{y}=B_{y}=0$, and we assume that all plasma variables are invariant in the $y$-direction, $\partial / \partial y=0$. This assumption results in a removal of the Alfvén wave from the system, which is able to propagate fast and slow magnetoacoustic waves only.

We consider an initial state, which corresponds to a straight slab model with an enhanced mass density over a width $a$ in an otherwise uniform and still medium (Nakariakov et al. 1995). The magnetic field is uniform and directed along the $x$-direction, $\boldsymbol{B}_{0}=B_{0} \hat{\boldsymbol{x}}$, where $\hat{\boldsymbol{x}}$ is a unit vector. The initial plasma flow, $\boldsymbol{V}_{0}=$ $V_{0}(x) \hat{\boldsymbol{x}}$, is limited to the slab only. This initial state is given by

$p, \varrho(z), B \hat{\boldsymbol{x}}, V(z) \hat{\boldsymbol{x}}= \begin{cases}p_{0}, \varrho_{\mathrm{i}}, B_{0}, V_{0}(x), & |z| \leq a, \\ p_{0}, \varrho_{\mathrm{e}}, B_{0}, 0, & |z|>a\end{cases}$

here the width of the slab, $a$, is chosen equal to $2.5 \mathrm{Mm}$. We take the mass density within the slab, $\varrho_{i}$, to be 5 times larger than in the ambient medium, $d=\varrho_{\mathrm{i}} / \varrho_{\mathrm{e}}=5$. This value is consistent with the observational data of Aschwanden \& Nightingale (2005). Additionally, we implement dense plasma layers for $x<x_{1}=0.15 L$ and $x>x_{\mathrm{r}}=0.76 \mathrm{~L}$, where $L=100 \mathrm{Mm}$ is the system length. This is realised by setting mass density $\tilde{\varrho}_{\mathrm{e}}(x, z)$ :

$$
\begin{aligned}
\tilde{\mathrm{e}}_{\mathrm{e}}(x, z)= & \varrho_{\mathrm{e}}(z)+\frac{1}{2} \varrho_{\mathrm{e}}(z)\left(d_{\mathrm{ph}}-1\right)\left\{\left[1-\tanh \left(\frac{x-x_{\mathrm{l}}}{s_{\mathrm{ph}}}\right)\right]\right. \\
& \left.+\left[1-\tanh \left(\frac{x-x_{\mathrm{r}}}{s_{\mathrm{ph}}}\right)\right]\right\} .
\end{aligned}
$$

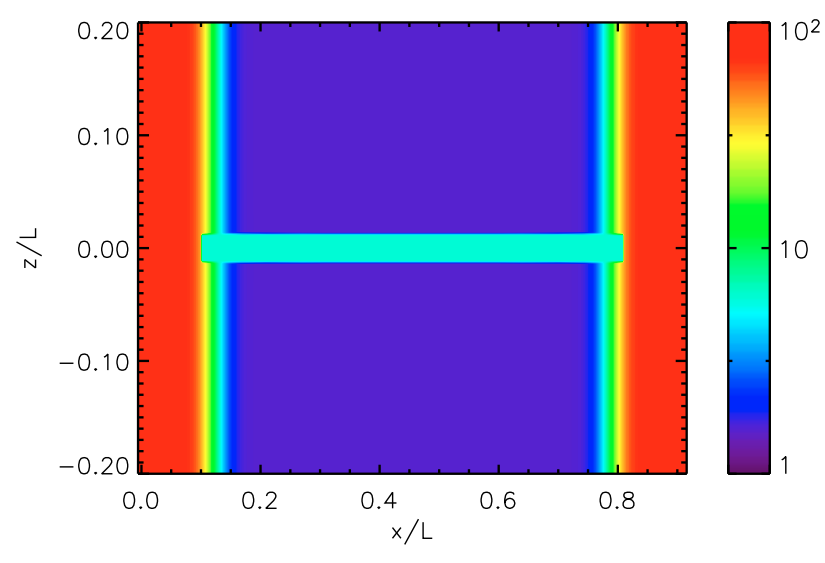

Fig. 1. Equilibrium mass density profile representing a straight coronal slab. Note dense photosphere layers for $x<0.15 L$ and $x>0.76 L$.

Here $x_{1}=0.15 L$ and $x_{\mathrm{r}}=0.76 L$ denote left and right positions of the dense layers, which give the length of the slab $L_{\mathrm{s}}=71 \mathrm{Mm}$. The ratio of the mass density of the photosphere to the ambient medium is chosen as $d_{\mathrm{ph}}=10^{2}$ and $s_{\mathrm{ph}}=2 \mathrm{Mm}$ is the width of the transition region. The dense layers represent the photosphere in which the slab footpoints are rooted. Initial configurations of the slab and the photosphere layers are shown in Fig. 1. The plasma flow of Eq. (7) is chosen either uniform within the slab

$V_{0}(x, z, t=0)=\hat{V}_{0}=$ const., $x_{1} \leq x \leq x_{\mathrm{r}},|z| \leq a$,

or varying along the slab according to the following formula:

$V_{0}(x, z, t=0)=\hat{V}_{0} \mathrm{e}^{-(x-L / 2)^{2} / \omega_{\mathrm{x}}^{2}},|z| \leq a$.

Here $\hat{V}_{0}$ denotes magnitude of the flow, $\hat{V}_{0}=92 \mathrm{~km} \mathrm{~s}^{-1}$. This value is close to the Hinode's observational data (Ofman \& Wang 2008). The symbol $\omega_{x}$ is the half-width of the initial flow profile. These two types of initially set flow are displayed in Fig. 2.

It is noteworthy here that the above described mass density and flow do not correspond to any exact equilibrium. Even initially uniform flow within the slab suffers from the lack of equilibrium that results from flux $\tilde{\varrho}_{\mathrm{e}} \boldsymbol{V}_{0}$, which is not conserved at $x=x_{1}$ and $x=x_{\mathrm{r}}$. The dense photosphere layers serve as natural wave reflectors. As they mimic action of the photosphere they consist of necessary ingredients of the developed model. Note that, as a flow magnitude $\hat{V}_{0}$ is much smaller than Alfvén speed in the ambient coronal medium $c_{\mathrm{A}}=\sqrt{B_{0} / \mu \varrho_{\mathrm{e}}}$ the initial state evolves on a slow temporal scale. This evolution results from plasma reflection from the dense plasma layers, but the system remains at the initial stage of its evolution in a quasi-equilibrium.

We choose and hold fixed $c_{\mathrm{A}}=10^{6} \mathrm{~m} \mathrm{~s}^{-1}$ and $c_{\mathrm{se}}=$ $\sqrt{\gamma p_{0} / \varrho_{\mathrm{e}}}=2 \times 10^{5} \mathrm{~m} \mathrm{~s}^{-1}$ for the sound speed in the ambient coronal medium. As a result, plasma beta $\beta=2 \mu p_{0} / B_{0}^{2}=0.048$ in the overall region. For this choice of parameters sound speed in the slab $c_{\mathrm{si}}=\sqrt{\gamma p_{0} / \varrho_{\mathrm{i}}}=0.92 \times 10^{5} \mathrm{~m} \mathrm{~s}^{-1}$. This value of $c_{\mathrm{si}}$ is close to the detected flow speed in the Hinode's data (Ofman \& Wang 2008).

\section{Numerical results}

To obtain numerical results we use the code ATHENA as described by Gardiner \& Stone (2005). ATHENA is a grid 


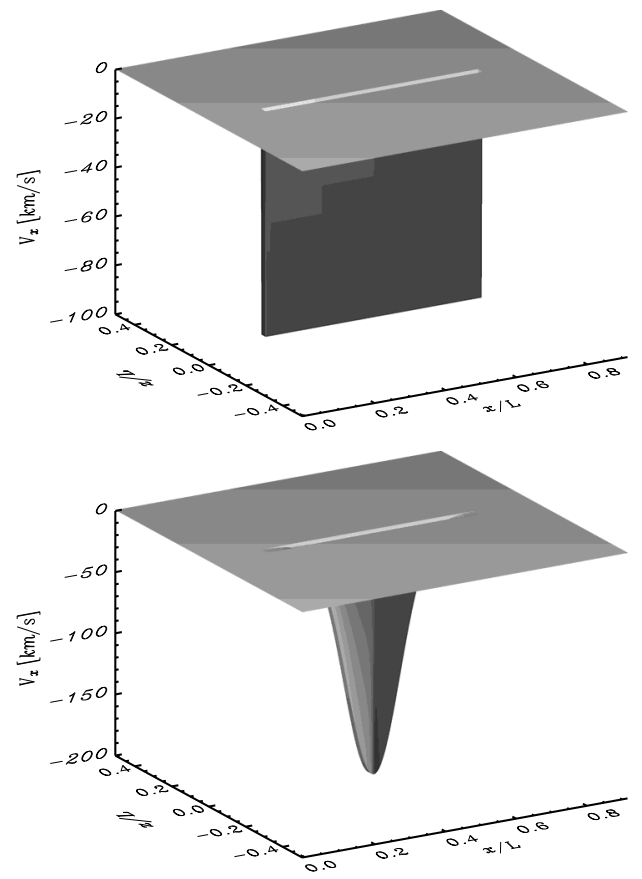

Fig. 2. Two types of field-aligned flow, initially launched within the slab: uniform flow (top panel) and Gaussian flow (bottom panel).

based code that is developed for astrophysical plasma dynamics applications. This code implements the higher-order Godunov method. Numerical fluxes are computed using a linearised Riemann solver and the divergence-free condition is satisfied with the use of a constrained transport method. To represent a physical region we use an Eulerian box $(0,0.91 L) \times$ $(-0.5 L, 0.5 L)$. This box is covered by $400 \times 400$ grid points. We set open boundary conditions at all boundaries of the simulation region, allowing an outgoing wave signal to leave freely the simulation area.

Fast magnetoacoustic kink oscillations are triggered impulsively by launching the initial pulse in vertical component of velocity $V_{z}$, i.e.

$V_{z}(x, z, t=0)=V_{z 0} \quad \exp \left[-\frac{\left(x-x_{0}\right)^{2}+\left(z-z_{0}\right)^{2}}{w^{2}}\right]$.

Here $x_{0}$ and $z_{0}$ denote the pulse's initial position, $w=10 \mathrm{Mm}$ is a parameter that determines its width and $V_{z 0}=0.092 c_{\mathrm{A}}$ is its amplitude. As we aim to excite the fundamental kink mode we launch the pulse centrally and below the slab. In particular, we set $x_{0} / L=0.455$ and $z_{0} / L=-0.1$. This pulse results in a wave signal that hits the slab centre, at $x / L=0.42$.

Figure 3 displays contour lines of $\varrho(x, z)$, evaluated at two consecutive times. Such lines denote two spatial positions of the slab, which are approximately in anti-phase. We infer from these positions that the slab essentially oscillates at the fundamental fast magnetoacoustic kink mode.

Figure 4 illustrates time-signatures of mass density evaluated at the slab for still (top panel) and initially uniform flowing (bottom panel) plasmas. Kink oscillations are clearly seen in both panels. Additionally, bottom panel reveals slow magnetoacoustic sausage oscillations, which result from reflected plasma from the left photosphere layer. These oscillations reach the detection region at $t \approx 200 \mathrm{~s}$, the moment at which mass density falls off abruptly. The rarefaction of the slab is associated with the Bernoulli effect. At a place where a gas pressure, magnetic pressure, and flow attain high values, a plasma within the slab has

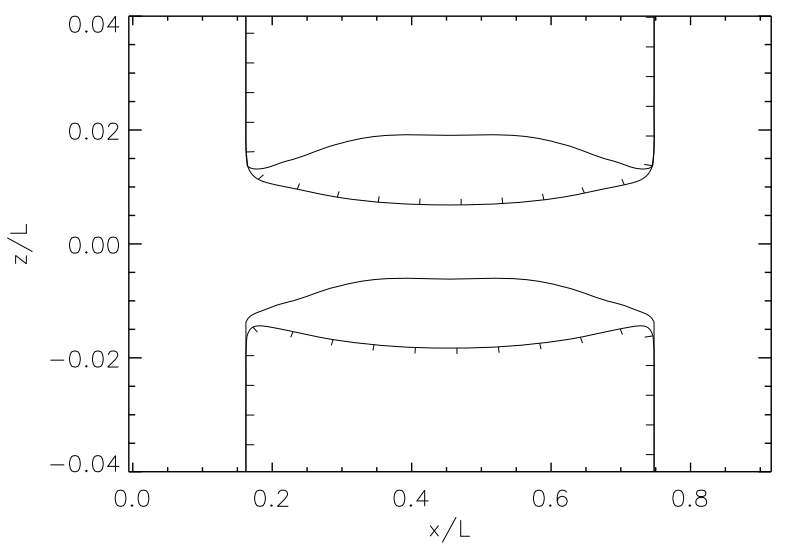

Fig. 3. Spatial position of the slab and photosphere edges at $t=25 \mathrm{~s}$ (solid lines) and $t=100 \mathrm{~s}$ (ticked lines) for the case of still plasma.
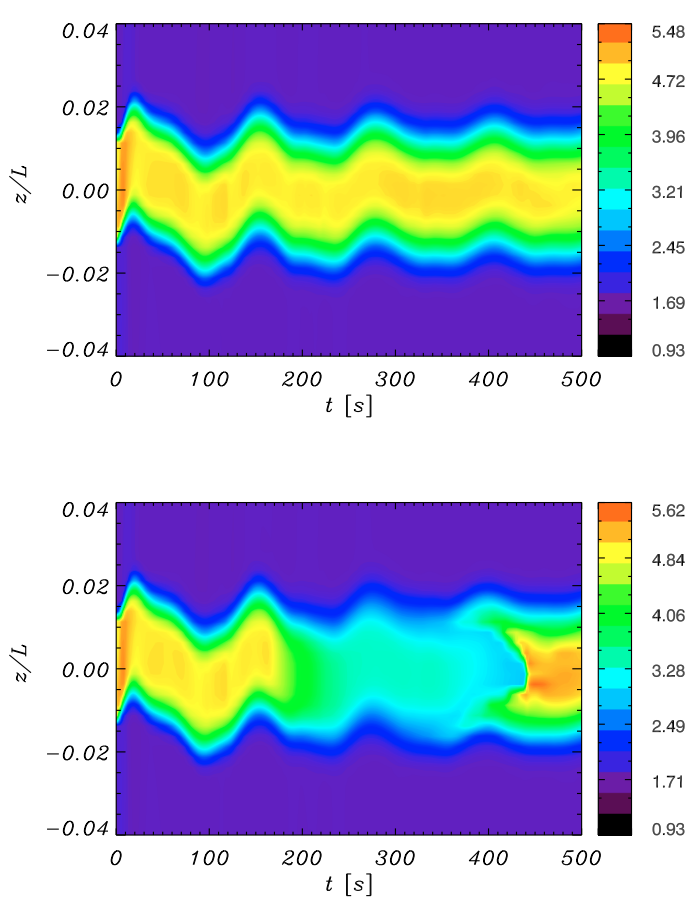

Fig. 4. Time-signatures of mass density evaluated at $x / L=0.42$ for still (top panel) and initially (at $t=0 \mathrm{~s}$ ) uniform flowing (bottom panel) plasmas.

to be rarefied to satisfy the Bernoulli equation, which is valid for a stationary flow. For an incompressible plasma this equation stands as

$\frac{p}{\gamma-1}+\frac{B^{2}}{2 \mu}+\frac{\varrho V^{2}}{2}=$ const.

From Fig. 4, we can see that at time $t=200-400 \mathrm{~s}$ the slab is rarefied the most. As a result, we conclude that the slab-aligned flow exerts a strong influence on slab mass density, leading to significant rarefaction of the slab at a location of a shock that is associated with the slow magnetoacoustic waves.

Figure 5 shows positions of the slab centre for the case of initially still plasma (solid line), uniform flow (dashed line), and Gaussian flow (dotted line). It is noteworthy that maximum shifts for still and flowing plasmas are of similar magnitude 

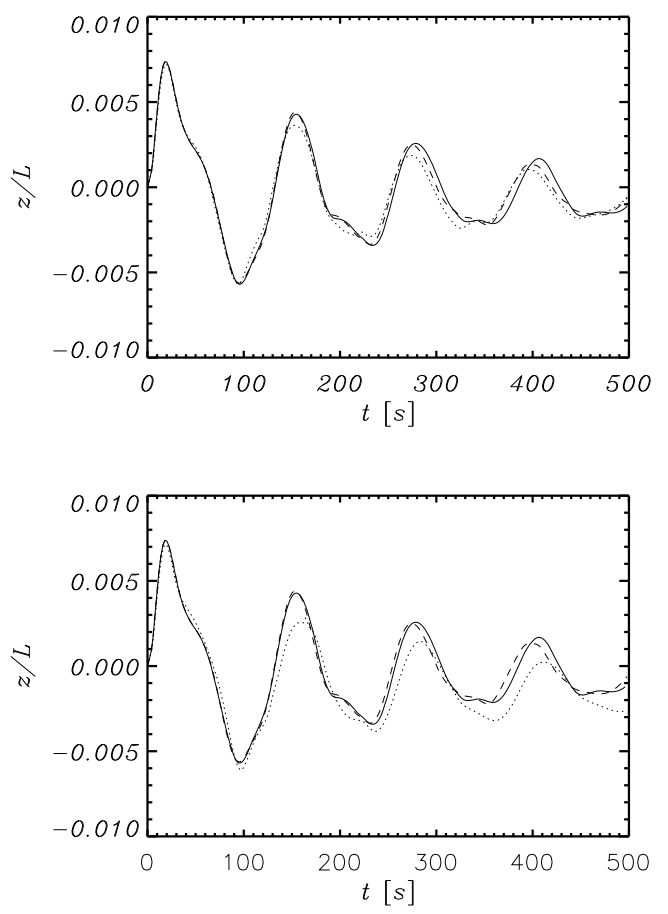

Fig. 5. A position of the slab centre evaluated from Fig. 4; solid line corresponds to $\hat{V}_{0}=0$, dashed line to $\hat{V}_{0}=92 \mathrm{~km} \mathrm{~s}^{-1}$ and dotted line to Gaussian flow with $w_{\mathrm{x}}=15 \mathrm{Mm}$ (top panel) and $w_{\mathrm{x}}=10 \mathrm{Mm}$ (bottom panel).

( $\left.A_{\max }=0.73 \mathrm{Mm}\right)$, which is close to the observational data of Hinode, $A_{\max }=0.67 \pm 0.12 \mathrm{Mm}$ (Ofman \& Wang 2008). Up to $t \simeq 200 \mathrm{~s}$, the slab positions essentially overlap each other for still and flowing plasmas, suggesting that flowing plasma does not alter significantly time-signatures of fast magnetoacoustic kink waves. At a later stage, some differences rise up and they grow up in time. At this stage, initially uniform flowing plasma becomes inhomogeneous, essentially as a result of reflection from the left dense layer. This partial reflection results in wave attenuation, which is further enhanced by wave scattering from inhomogeneities. As a result of this scattering, fast magnetoacoustic waves reduce their amplitude and alter their wave period. Such phenomena are well known for waves in randomly flowing fluid (e.g., Murawski 2004). We infer that the slab oscillations are attenuated at a higher rate for flowing plasma than for still plasma.

From Fig. 5 we can estimate wave period $P$ and attenuation time $\tau$. For this purpose we fit a position of the slab centre to the attenuated sine function $\sin (2 \pi t / P) \exp (-t / \tau)($ e.g., Gruszecki et al. 2008). We find that for the case of initially uniform flowing plasma $P=124 \mathrm{~s}$, which is somewhat lower than for still plasma $(P=126 \mathrm{~s})$. These values are close to the detected wave period by Hinode $P=113 \pm 2$ s (Ofman \& Wang 2008). This wave period reduction may result from Doppler effect from rightward directed flow, which settles in the system after the original flow reflection from the left photosphere layer. Note that the difference in the estimated wave periods of the long-wavelength kink mode in the tube and slab geometry. In the long-wavelength limit, the phase speed of this mode tends to the external Alfvén speed in the slab case, while it tends to the kink speed in the tube case. This clearly leads to the error in the theoretical estimation of the phase speed and, as a result, in a wave period.
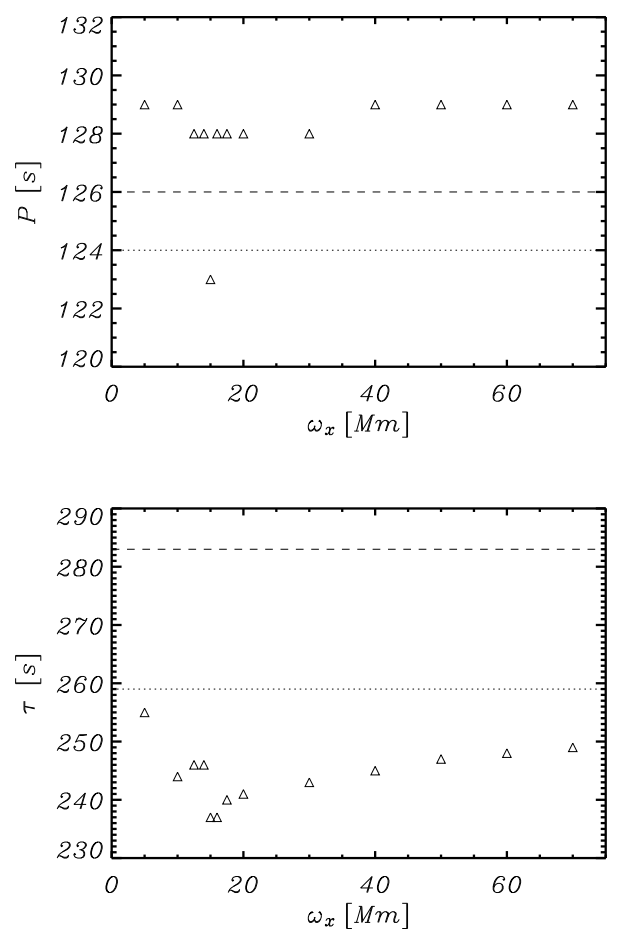

Fig. 6. (Top) Wave period $P$ vs. half-width of the initial Gaussian flow $\omega_{x}$. (Bottom) Attenuation time $\tau$ vs. $\omega_{x}$. Horizontal dashed lines correspond to the data for still $\left(V_{0}=0\right)$ and dotted line to flowing $\left(V_{0}=\right.$ const. $)$ plasmas.

We find that attenuation times differ for the case of still plasma and for the case of flowing plasma; for still plasma we get $\tau=283 \mathrm{~s}$ and for uniform flowing plasma we obtain $\tau=259 \mathrm{~s}$. From the obtained results we infer that the oscillations are stronger attenuated for the flowing plasma case. This stronger attenuation results from wave scattering on plasma inhomogeneities, which become more pronounced for initially inhomogeneous flow and when flowing plasma reflects from the region $x \approx 0.15 \mathrm{~L}$. As a consequence of this reflection even initially homogeneous flow becomes inhomogeneous and a slow magnetoacoustic sausage wave settles in.

To make a deeper analysis of the effect of initial flow $V_{0}$ on $P$ and $\tau$, we consider now Gaussian flow inside the slab (Fig. 2, bottom panel). We investigate the importance of the half-width of the initial flow profile, $\omega_{x}$, on the wave characteristics. We vary $\omega_{x}$ from $\omega_{x}=5 \mathrm{Mm}$ to $\omega_{x}=70 \mathrm{Mm}$. In the later case, the flow becomes essentially homogeneous. A value of flow amplitude $\hat{V}_{0}$ was chosen in a way that kinetic energy inside the slab remains initially invariant with $\omega_{x}$. The results of our simulations are shown in Fig. 6. From the obtained results we infer that, according to our expectations, $P$ remains weakly dependent on the initial flow. However, wave period is higher than in the case of still plasma. Both in the case of constant flow and Gaussian flow wave period is altered by less than $1 \%$, which is insignificant for coronal slab application. Wave period $P$ attains a minimum for $w_{x} \simeq 15 \mathrm{Mm}$.

Figure 6 (bottom panel) illustrates attenuation time $\tau$ vs. $\omega_{x}$. From the obtained results we infer that $\tau$ also reaches a minimum for $\omega_{x} \simeq 15 \mathrm{Mm}$. A physical reason for a location of the minimum is presently unclear. We might expect that for a very narrow Gaussian profile, $\omega_{x}$, the effect of flow is small on wave attenuation. Simply, in this case, wavelength (that is equal 
to $2 L_{\mathrm{S}}$ ) is so large that small-scale inhomogeneity is not important. Similarly, in the limit of large $\omega_{x}$ a fast magnetoacoustic wave perceives such a flow as locally constant and as a result the flow effect is small. A stronger effect of flow should occur for intermediate values of $\omega_{x}$. Indeed a strongest effect is expected when flow exhibits spatial scales, which are comparable with the wavelength.

Note, that Gruszecki et al. (2008) showed that $\tau$ depends on density contrast $d_{\mathrm{ph}}$ and smoothness $s_{\mathrm{ph}}$ of the dense photosphere-like layer. They estimated that $\tau / P$ grows by about a factor of 2 , while $d_{\mathrm{ph}}$ varies from $10^{2}$ to $10^{4}$. Thus, choosing a density contrast between photosphere and corona as $d_{\mathrm{ph}}=10^{4}$ we get closer to the observational value of $\tau=560 \pm 260 \mathrm{~s}$ (Ofman \& Wang 2008).

\section{Summary and discussion}

We discussed the effect of uniform and inhomogeneous initial flows on wave characteristics of fast magnetoacoustic kink oscillations of the fundamental standing mode. Our findings can be summarised as follows: neither uniform nor inhomogeneous flows are able to alter significantly wave period of the mode. Note that Murawski (2004) found that a randomly structured flow leads to frequency reduction (wave period increase) of sound waves. However, this result was obtained for an ensemble averaged random field and for some realisations of this field wave frequency increase was reported. Whether wave frequency is decreased or increased depends on wave scattering scenario on random inhomogeneities. As we got that $P$ is decreased by Gaussian flow our findings fit into findings for those particular realisations of a random field, for which $P$ is reduced (Murawski 2004). As a result of wave scattering on an inhomogeneous flow a stronger wave attenuation is also present in the case of random flow (Murawski 2004). This attenuation was obviously strongest for the case of a most abruptly varying spatial profile of the flow; for Gaussian flow with $\omega_{x}=15 \mathrm{Mm}$ attenuation time $\tau$ was reduced by $\sim 14 \%$ in comparison to the case of still plasma. It is noteworthy that flow results in Doppler shift. The influence of the flow on the wavelength of the fast mode is small since flow speed $V_{0} \ll c_{\mathrm{A}}$. However, $V_{0}$ is comparable to a typical wave speed of a slow magnetoacoustic wave, and can effect the slow magnetoacoustic wave frequency due to Doppler shift.

This study concludes that the flow increases the wave attenuation. However, in the Hinode observation very weak attenuation is seen (Ofman \& Wang 2008). One of possible ways to remedy this is to consider negative energy waves (i.e., the flow supplies energy to the waves). Studies by Soler et al. (2008) are relevant in this case. Finally, we mention that the agreement of our numerical model with Hinode observations of Ofman \& Wang (2008) as well as with the theory of coronal seismology (Nakariakov \& Verwichte 2005) is encouraging.

It is noteworthy that the slow shock (Fig. 4, bottom panel) is not seen in the loop in Hinode observations (Ofman \& Wang 2008). This shock is due to the cold slab model we have chosen, and the reflections which occur at the photosphere-like layers. Since the goal of the paper was to compare to the Hinode data it is important to remove this inherent feature. One way of doing so is a construction of an isothermal slab model (Murawski et al. 1997). In this model a uniform parallel flow is set along a warm

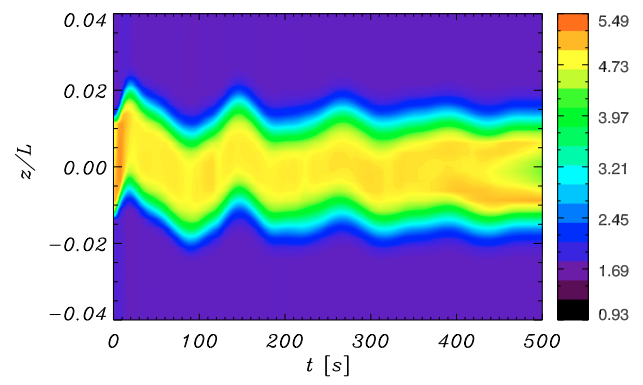

Fig. 7. Time-signatures of mass density evaluated at centre of the slab.

slab. In this slab internal magnetic field $B_{\mathrm{i}}$ (gas pressure $p_{\mathrm{i}}$ ) within the slab is lower (higher) than in the ambient medium. In particular we have chosen

$\frac{B_{\mathrm{e}}}{B_{\mathrm{i}}}=1.12, \quad \frac{p_{\mathrm{i}}}{p_{\mathrm{e}}}=5, \quad \frac{T_{\mathrm{e}}}{T_{\mathrm{i}}}=1$.

Figure 7 illustrates time-signature of the mass density collected at the slab centre. According to our expectations a slow shock is absent in this time-signature and therefore such a model would be more realistic.

Acknowledgements. The authors express their thanks to the referee for his/her stimulating comment. M.G.'s and K.M.'s work was supported by the grant for years 2007-2010 from the Polish Ministry of Science. L.O. is supported by NASA Sun-Earth Connection theory program, NASA grant NNG06GI55G, and NRL grant N00173-06-1-G033. Athena is a grid-based code for astrophysical gas dynamics being developed with support of the NSF Information Technology Research (ITR) program. The magnetohydrodynamics code used in this study was developed at the Princeton University by Tom Gardiner, Jim Stone, Peter Teuben and John Hawley with support of the NSF Information Technology Research program.

\section{References}

Aschwanden, M. J., \& Nightingale, R. W. 2005, ApJ, 633, 499

Aschwanden, M. J., Fletcher, L., Schrijver, C. J., \& Alexander, D. 1999, ApJ, 520,880

DeForest, C. E., \& Gurman, J. B. 1998, ApJ, 501, L217

De Moortel, I., Ireland, J., \& Walsh, R. W. 2002, TRACE Observations of propagating slow magneto-acoustic disturbances in coronal loops, ESA-SP-508, 275

Grappin, R., Léorat, J., \& Ofman, L. 2003, SOLAR WIND TEN, 679, 750

Grappin, R., Léorat, J., \& Habbal, S. R. 2005, A\&A, 437, 1081

Gruszecki, M., Murawski, K., \& McLaughlin, J. A. 2008, A\&A, in press

Joarder, P. S., Nakariakov, V. M., \& Roberts, B. 1997, Sol. Phys., 176, 285

Murawski, K. 2002, Analytical and numerical methods for wave propagation in fluids (World Scientific)

Nakariakov, V. M., \& Roberts, B. 1995, Sol. Phys., 159, 213

Nakariakov, V. M., \& Verwichte, E. 2005, Liv. Rev. Sol. Phys., 2, 3

Ofman, L., \& Wang, T. J. 2008, A\&A, 482, L9

Ofman, L., Romoli, M., Poletto, G., Noci G., \& Kohl, J. L. 1997, ApJ, 491, L111

Ofman, L., Nakariakov, V. M., \& DeForest, C. E. 1999, ApJ, 514, 441

Roberts, B., Edwin, P. M., \& Benz, A. O. 1984, ApJ, 279, 857

Soler, R., Oliver, R., \& Ballester, J. L. 2008, ApJ, submitted

Terra-Hommem, M., Erdélyi, R., \& Ballai, I. 2003, Sol. Phys., 217, 199

Uchida, Y. 1970, PASJ, 22, 341

Wang, T. J., \& Solanki, S. K. 2004, A\&A, 421, L33

Wang, T. J., Solanki, S. K., Curdt, W., Innes, D. E., \& Dammash, I. E. 2002, ApJ, 574, L101 